J. Korean Math. Soc. 47 (2010), No. 5, pp. 925-934

DOI 10.4134/JKMS.2010.47.5.925

\title{
REGULAR GENUS AND PRODUCTS OF SPHERES
}

\author{
Fulvia Spaggiari
}

\begin{abstract}
A crystallization of a closed connected PL manifold $M$ is a special edge-colored graph representing $M$ via a contracted triangulation. The regular genus of $M$ is the minimum genus of a closed connected surface into which a crystallization of $M$ regularly embeds. We disprove a conjecture on the regular genus of $\mathbb{S}^{2} \times \mathbb{S}^{n}, n \geq 3$, stated in [J. Korean Math. Soc. 41 (2004), no. 3, p. 420].
\end{abstract}

\section{Introduction}

Throughout the paper we shall work in the piecewise linear (PL) category. For basic definitions and results on PL topology see, for example, [14] and [17]. An $(n+1)$-colored graph is a pair $(G, c)$, where $G=(V(G), E(G))$ is a connected multigraph (without loops) regular of degree $n+1$, and $c: E(G) \rightarrow$ $\Delta_{n}=\{0,1, \ldots, n\}$ is an edge-coloring such that $c(e) \neq c(f)$ for any pair $e$, $f$ of adjacent edges of $G$. The set $\Delta_{n}$ will be called the color set and its elements the colors. Colored graphs represent a useful combinatorial approach to the topology of piecewise linear (PL) manifolds. For more details see for example the survey papers [1], [5], [7], [10], and [18]. An n-pseudocomplex $K(G)$ can be associated to $(G, c)$ by the following rules. We consider an $n$ simplex $\sigma^{n}(v)$ for each vertex $v$ of $G$ and label its vertices by $\Delta_{n}$. If two vertices $v$ and $w$ are joined in $G$ by an $i$-colored edge, then we identify the $(n-1)$-faces of the simplexes $\sigma^{n}(v)$ and $\sigma^{n}(w)$ opposite to the vertex labelled by $i$, so that equally labelled vertices are identified. For each $B \subset \Delta_{n}$, the set $G_{B}=\left(V(G), c^{-1}(B)\right)$ is a partial subgraph of $G$ and its connected components are called the $B$-residues of $G$. If $B=\{i, j\} \subset \Delta_{n}$, let $g_{i j}$ denote the number of $B$-residues in $G$. For each color $i \in \Delta_{n}$, we set $\widehat{i}=\Delta_{n} \backslash\{i\}$. Then $(G, c)$ is said to be contracted if $G_{\hat{i}}$ is connected for every $i \in \Delta_{n}$. An $(n+1)$-colored graph $(G, c)$ is said to be a crystallization of a closed connected PL $n$-manifold $M$ if $(G, c)$ is contracted and the polyhedron $|K(G)|$, underlying $K(G)$, is (PL) homeomorphic to $M$. We say that $K(G)$ is a contracted triangulation of $M$

Received October 16, 2008.

2000 Mathematics Subject Classification. 57M15, 57Q15, 05C10.

Key words and phrases. PL manifold, regular genus, product of spheres, crystallization. 
and that $G$ represents $M$. It is well known that any closed connected PL $n$ manifold admits a crystallization (see [15] and [16]). Any two colored-graphs (in particular crystallizations) which represent the same manifold are related by a finite sequence of certain elementary moves, called cancelling and/or adding dipoles (see [9]). A subgraph $\Theta$ of $G$ formed by two vertices $x$ and $y$, joined by $h$ edges, $1 \leq h \leq n$, labelled by colors $i_{1}, i_{2}, \ldots, i_{h}$, is called a dipole of type $h$ if $x$ and $y$ belong to different connected components of the partial subgraph $G_{\Delta_{n} \backslash\left\{i_{1}, i_{2}, \ldots, i_{h}\right\}}$. Cancelling $\Theta$ means the following two steps: (1) delete $x$ and $y$ together with the $h$ edges joining them; (2) paste the pairs of dangling edges of the same color. Adding $\Theta$ means the inverse process. A 2-cell embedding $f:|G| \rightarrow F$ of an $(n+1)$-colored graph into a closed connected surface $F$ is called regular if there exists a cyclic permutation $\epsilon=\left(\epsilon_{0}, \epsilon_{1}, \ldots, \epsilon_{n}\right)$ of $\Delta_{n}$ such that each region of $f$ is bounded by a cycle with edges alternatively colored by $\epsilon_{i}, \epsilon_{i+1}$ (indices $\left.\bmod n+1\right)$. The Euler characteristic of $F$ (see [11]) is

$$
\chi(F)=\sum_{i \in \mathbb{Z}_{n+1}} g_{\epsilon_{i} \epsilon_{i+1}}+\frac{(1-n)}{2} p,
$$

where $g_{\epsilon_{i} \epsilon_{i+1}}$ is the number of $\left\{\epsilon_{i}, \epsilon_{i+1}\right\}$-residues of $G$ and $p$ is the number of vertices of $G$ (i.e., the order of $G$ ). The surface $F$ is orientable if and only if $G$ is bipartite. The regular genus $g(G)$ of $G$ is the minimum genus of a closed connected surface into which $G$ regularly embeds. The regular genus $g(M)$ of a closed connected PL $n$-manifold $M$ was defined in [12] as the integer

$$
g(M)=\min \{g(G):(G, c) \text { is a crystallization of } M\} .
$$

This topological invariant extends to dimension $n$ the classical notions of genus of a closed surface and of Heegaard genus of a closed 3-manifold. It is known that the $n$-sphere $\mathbb{S}^{n}$ is the unique closed connected PL $n$-manifold of regular genus zero. There are a lot of results on the computation of the regular genus for closed PL manifolds (see, for example, the survey papers quoted above, and their references). Here we are interested in the case of products of spheres. The following facts are known:

- The product $\mathbb{S}^{1} \times \mathbb{S}^{n}, n \geq 3$, is the unique closed connected PL $(n+1)$ manifold of regular genus one $([3],[6])$.

- The regular genus of $\mathbb{S}^{2} \times \mathbb{S}^{2}$ (resp. $\mathbb{S}^{2} \times \mathbb{S}^{3}$ ) is four (resp. eight) ([2], [4]).

- For each $n \geq 3, g\left(\mathbb{S}^{2} \times \mathbb{S}^{n}\right) \leq n^{2}-1([8])$.

The following conjecture was stated in [8], p. 420.

Conjecture. For each $n \geq 3, g\left(\mathbb{S}^{2} \times \mathbb{S}^{n}\right)=n^{2}-1$.

This is true for $n=3$. In this paper we show that the conjecture is false for $n=4$ and $n=5$. In fact, we have: 
Theorem. There are crystallizations of $\mathbb{S}^{4} \times \mathbb{S}^{2}$ and $\mathbb{S}^{5} \times \mathbb{S}^{2}$ which regularly embed into closed connected orientable surfaces of genus 13 and 19, respectively, so $g\left(\mathbb{S}^{4} \times \mathbb{S}^{2}\right) \leq 13$ and $g\left(\mathbb{S}^{5} \times \mathbb{S}^{2}\right) \leq 19$.

\section{Representing products of spheres by colored graphs}

A recursive algorithm for constructing noncontracted colored graphs representing products of spheres was given in [8]. Such a construction is the first step we use to obtain contracted colored graphs (i.e., crystallizations) representing $\mathbb{S}^{4} \times \mathbb{S}^{2}$ and $\mathbb{S}^{5} \times \mathbb{S}^{2}$. To make the reading of the paper self-contained, we briefly recall the algorithm described in [8]. It is based on the standard $(p+1)$-colored graph $G^{(p)}$ with two vertices joined by $p+1$ differently colored edges, which represents the $p$-sphere $\mathbb{S}^{p}$. An $(m+n+1)$-colored graph $G^{(m, n)}$ with $4\left(\begin{array}{c}m+n \\ n\end{array}\right)$ vertices, representing the topological product $\mathbb{S}^{m} \times \mathbb{S}^{n}$, can be constructed in the following way:

1) The vertex set is denoted by

$$
V\left(G^{(m, n)}\right)=V\left(G^{(m-1, n)}\right) \cup V\left(G^{(m, n-1)}\right),
$$

where

$$
V\left(G^{(m-1, n)}\right)=\left\{\bar{\delta}_{j}^{i}(k): i, j=1,2, k=1, \ldots,(\underset{n}{m+n-1})\right\}
$$

and

$$
V\left(G^{(m, n-1)}\right)=\left\{\overline{\bar{\delta}}_{j}^{i}(k): i, j=1,2, k=1, \ldots,(\underset{m}{m+n-1})\right\} ;
$$

2) For each $k=1, \ldots,\left(\begin{array}{c}m+n-1 \\ n\end{array}\right)$, join $\bar{\delta}_{1}^{1}(k)$ with $\bar{\delta}_{1}^{2}(k)$ and $\bar{\delta}_{2}^{2}(k)$ with $\bar{\delta}_{2}^{1}(k)$ by an $(m+n)$-colored edge (the color set is $\left.\Delta_{m+n}\right)$;

3) For each $k=1, \ldots,\left(\begin{array}{c}m+n-1 \\ m\end{array}\right)$, join $\overline{\bar{\delta}}_{1}^{1}(k)$ with $\overline{\bar{\delta}}_{2}^{1}(k)$ and $\overline{\bar{\delta}}_{2}^{2}(k)$ with $\overline{\bar{\delta}}_{1}^{2}(k)$ by an $(m+n)$-colored edge;

4) For each $k=\left(\begin{array}{c}m+n-2 \\ n\end{array}\right)+1, \ldots,\left(\begin{array}{c}m+n-2 \\ n\end{array}\right)+\left(\begin{array}{c}m+n-2 \\ n-1\end{array}\right)$, join $\bar{\delta}_{j}^{i}(k)$ and $\overline{\bar{\delta}}_{j}^{i}(k-(\underset{n}{m+n-2})), i, j=1,2$, by an $(m+n-1)$-colored edge;

5) For the remaining vertices of $G^{(m, n)}$ re-establish the edges connecting them as they are in $G^{(m-1, n)}$ and $G^{(m, n-1)}$.

By this construction, starting from the $(p+2)$-colored graphs $G^{(1, p)}$ and $G^{(p, 1)}, p>0$, it is possible to construct the $(m+n+1)$-colored graph $G^{(m, n)}$ for each $m, n>0$. A picture of the graphs $G^{(1, n)}, n>0$, is shown in $[8$, Figure 3]. As remarked in [8], the graph $G^{(m, n)}$ has a double symmetry: each edge connecting the vertices $\bar{\delta}_{1}^{1}(k)$ and $\bar{\delta}_{1}^{2}(k)$ (resp. $\overline{\bar{\delta}}_{1}^{1}(k)$ with $\overline{\bar{\delta}}_{2}^{1}(k)$ ) has a corresponding edge, with the same color, connecting the vertices $\bar{\delta}_{2}^{2}(k)$ and $\bar{\delta}_{2}^{1}(k)\left(\right.$ resp. $\overline{\bar{\delta}}_{2}^{2}(k)$ with $\left.\overline{\bar{\delta}}_{1}^{2}(k)\right)$ for each $k=1, \ldots,\left(\begin{array}{c}m+n \\ n\end{array}\right)$.

In the next sections we consider the noncontracted colored graphs $G^{(4,2)}$ and $G^{(5,2)}$ representing $\mathbb{S}^{4} \times \mathbb{S}^{2}$ and $\mathbb{S}^{5} \times \mathbb{S}^{2}$, respectively. Cancelling successively 
many dipoles of types 1 and 2 on them, we obtain contracted colored graphs (that is, crystallizations) representing the same manifolds. Then we compute the genera of closed orientable surfaces into which such crystallizations regularly embed. This allows us to obtain new upper bounds for the regular genera of the above manifolds. Our bounds are stronger than those given in [8] for such products of spheres, and disprove the conjecture stated in that paper.

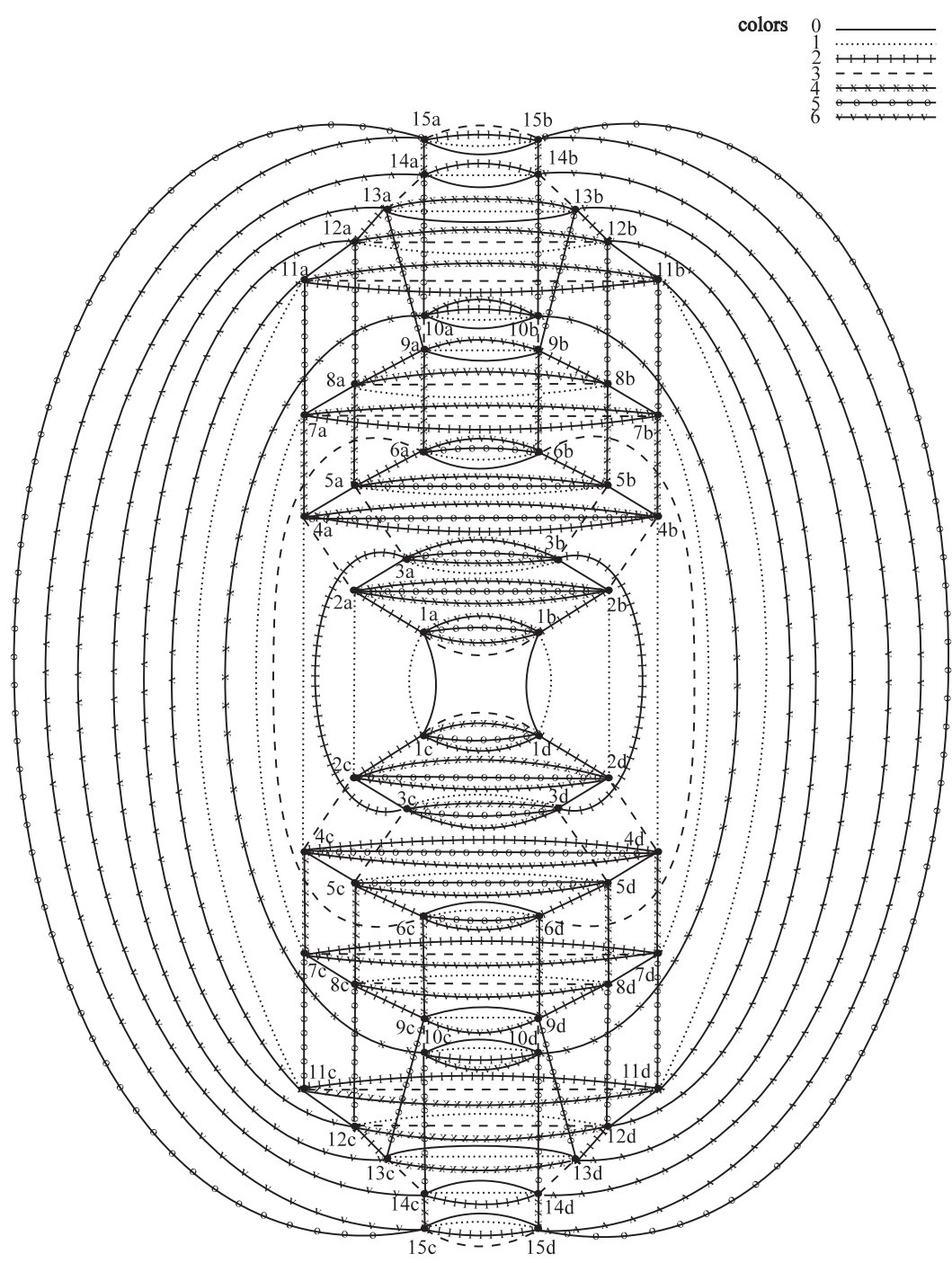

Figure 1. The 7-colored graph $G^{(4,2)}$ representing $\mathbb{S}^{4} \times \mathbb{S}^{2}$ 


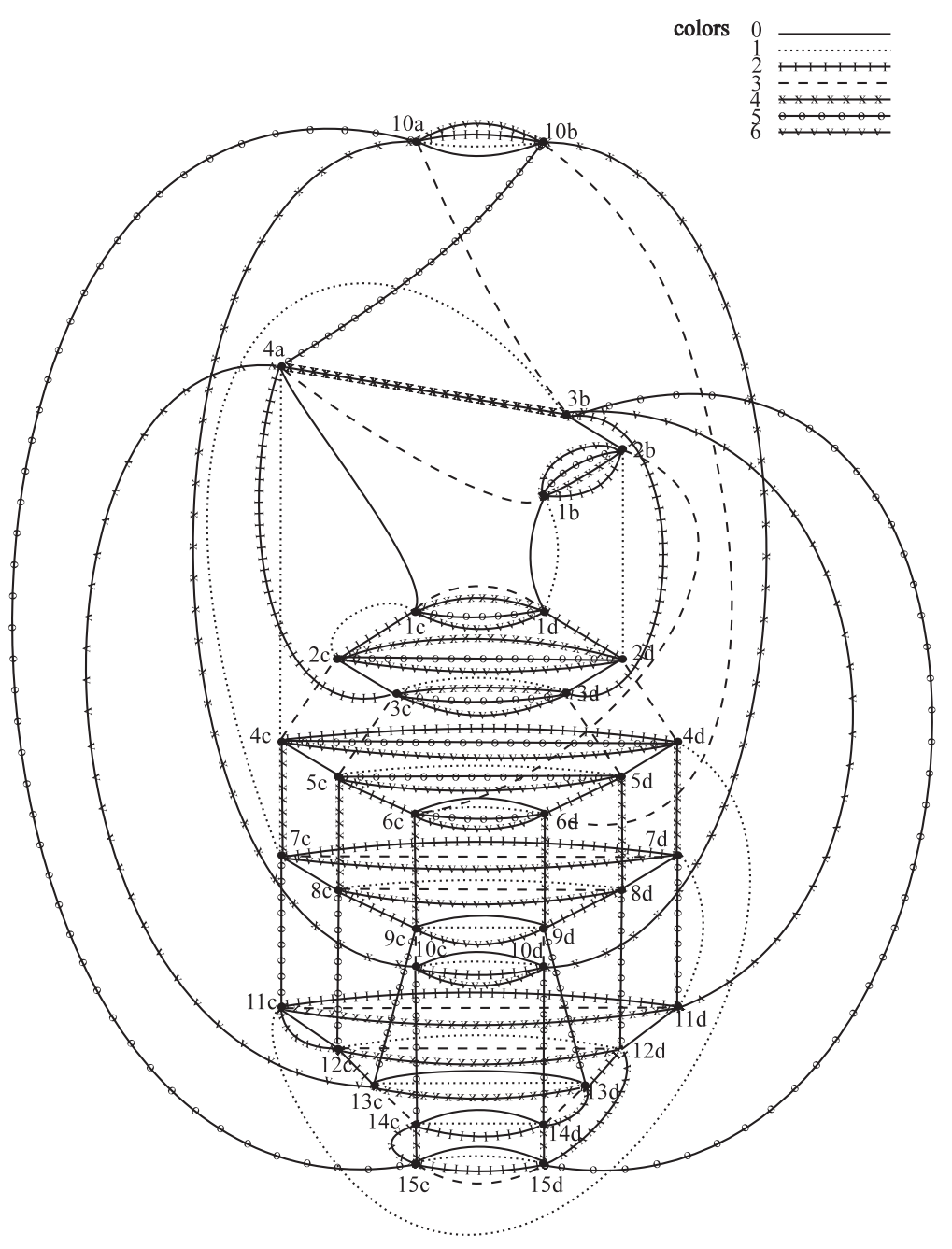

FIgURE 2. The crystallization $\widetilde{G}^{(4,2)}$ of $\mathbb{S}^{4} \times \mathbb{S}^{2}$

\section{A crystallization of $\mathbb{S}^{4} \times \mathbb{S}^{2}$}

We apply the above construction in order to obtain a 7-colored graph $G^{(4,2)}$ with 60 vertices representing the manifold $\mathbb{S}^{4} \times S^{2}$ (see Figure 1). To simplify the notation, the vertices $\bar{\delta}_{j}^{i}(k), i, j=1,2, k=1, \ldots,\left(\begin{array}{l}5 \\ 2\end{array}\right)=10$, are denoted in the following way: $\bar{\delta}_{1}^{1}(1)=1 a, \bar{\delta}_{1}^{2}(1)=1 b, \bar{\delta}_{2}^{1}(1)=1 c, \bar{\delta}_{2}^{2}(1)=1 d$, $\bar{\delta}_{1}^{1}(2)=2 a, \bar{\delta}_{1}^{2}(2)=2 b, \bar{\delta}_{2}^{1}(2)=2 c, \bar{\delta}_{2}^{2}(2)=2 d, \ldots, \bar{\delta}_{1}^{1}(10)=10 a, \bar{\delta}_{1}^{2}(10)=$ 


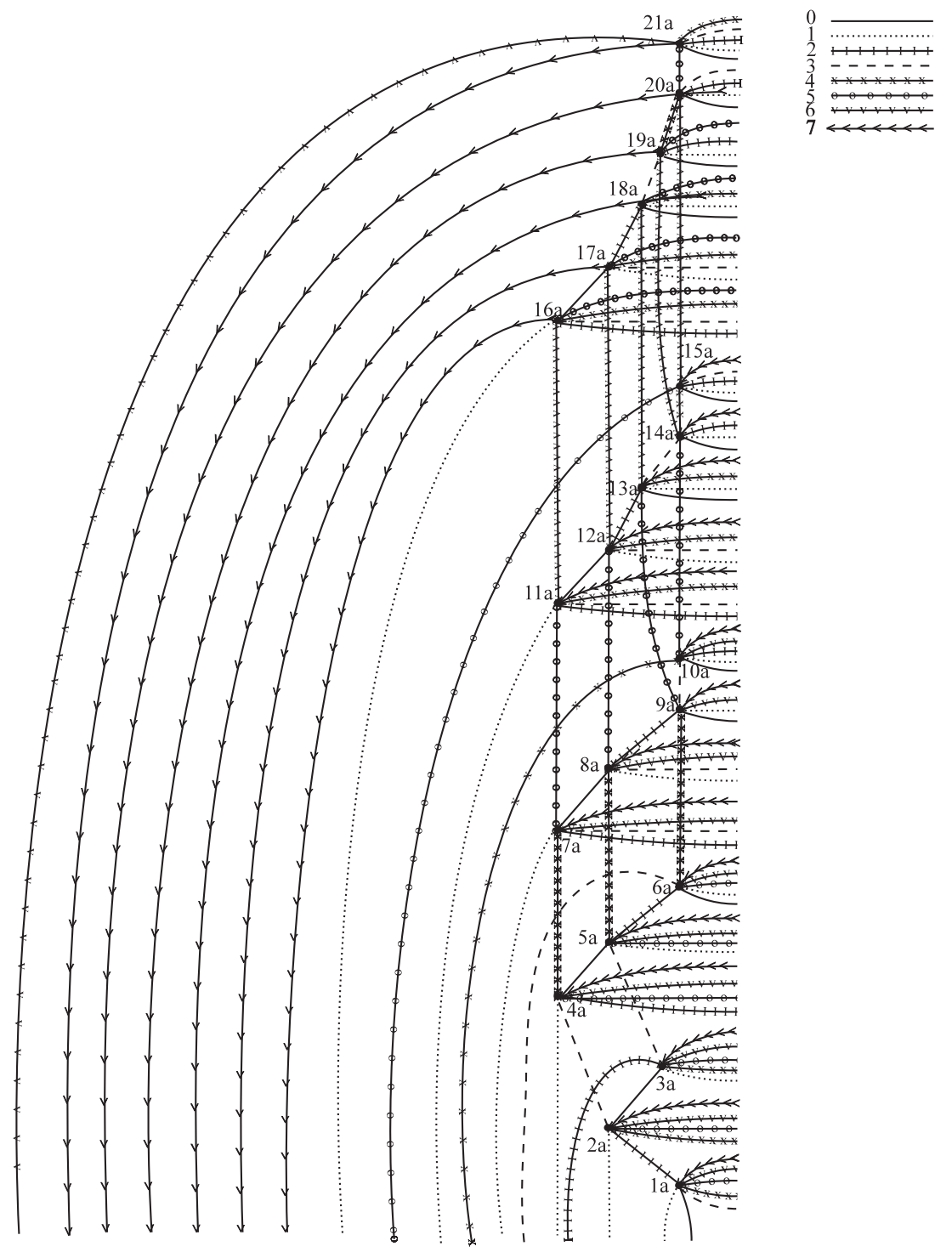

Figure 3. A part of the 8-colored graph $G^{(5,2)}$ representing $\mathbb{S}^{5} \times \mathbb{S}^{2}$

$10 b, \bar{\delta}_{2}^{1}(10)=10 c, \bar{\delta}_{2}^{2}(10)=10 d ;$ while the vertices $\overline{\bar{\delta}}_{j}^{i}(k), i, j=1,2, k=$ $1, \ldots,\left(\begin{array}{l}5 \\ 4\end{array}\right)=5$, are denoted as follows: $\overline{\bar{\delta}}_{1}^{1}(1)=11 a, \overline{\bar{\delta}}_{1}^{2}(1)=11 b, \overline{\bar{\delta}}_{2}^{1}(2)=11 c$, $\overline{\bar{\delta}}_{2}^{2}(1)=11 d, \ldots, \overline{\bar{\delta}}_{1}^{1}(5)=15 a, \overline{\bar{\delta}}_{1}^{2}(5)=15 b, \overline{\bar{\delta}}_{2}^{1}(5)=15 c, \overline{\bar{\delta}}_{2}^{2}(5)=15 d$. Then, 


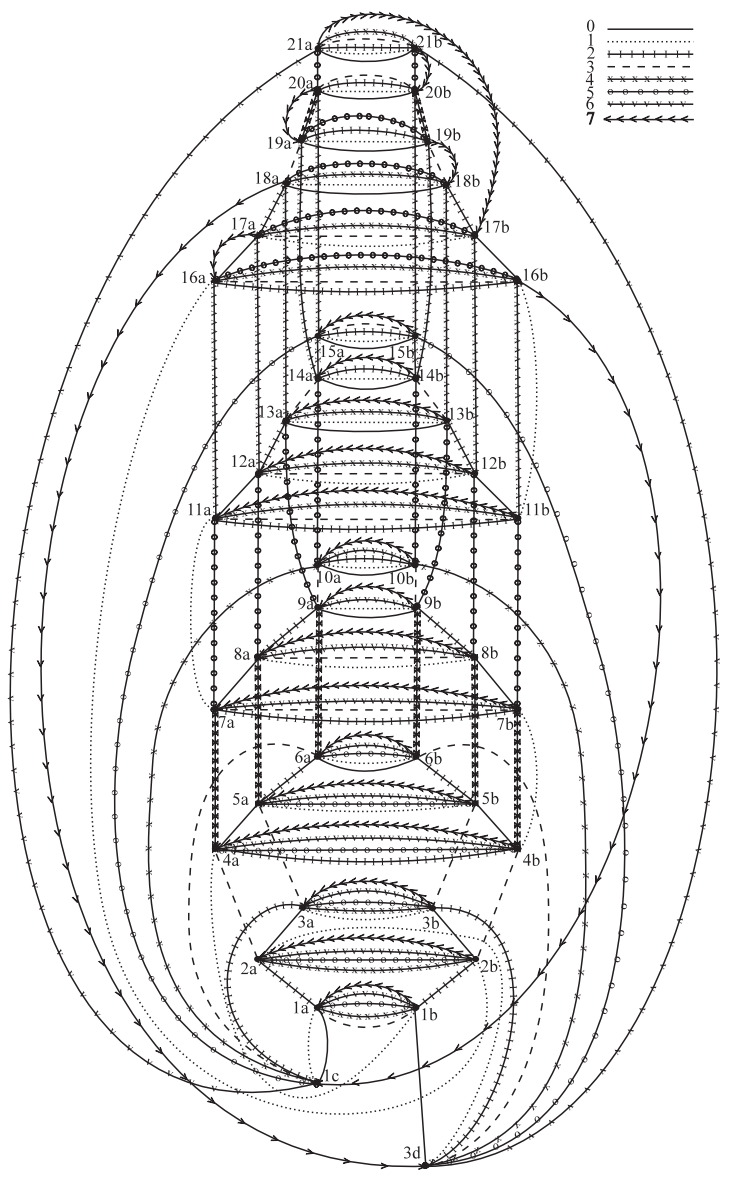

FIGURE 4 . The crystallization $\widetilde{G}^{(5,2)}$ of $\mathbb{S}^{5} \times \mathbb{S}^{2}$

according to steps (2) and (3) of the construction in Section 2, we join in pairs the vertices $1 a$ and $1 b$ (resp. $1 c$ and $1 d$ ), ..,10a and $10 b$ (resp. $10 c$ and $10 d$ ), and the vertices $11 a$ and $11 c$ (resp. $11 b$ and $11 d$ ), $\ldots, 15 a$ and $15 c$ (resp. $15 b$ and $15 d$ ) by 6 -colored edges. Finally, applying step (4), we join in pairs the vertices $7 a$ and $11 a$ (resp. $7 b, 7 c, 7 d$ with $11 b, 11 c, 11 d$ ), $\ldots, 10 a$ and $14 a$ (resp. 10b, 10c, 10d with $14 b, 14 c, 14 d$ ) by 5 -colored edges. For the remaining vertices of $G^{(4,2)}$ we consider the edges as they are in the graphs $G^{(3,2)}$ and $G^{(4,1)}$, representing $\mathbb{S}^{3} \times \mathbb{S}^{2}$ and $\mathbb{S}^{4} \times \mathbb{S}^{1}$, respectively, according to step (5). Now we simplify the graph $G^{(4,2)}$ by cancelling the dipole of type 1 formed by the vertices $15 a$ and $14 a$ joined by one edge labelled by color 
4 , as these vertices belong to different connected components of $G_{\widehat{4}}$. So we delete $15 a$ and $14 a$ together with the edge joining them and paste the pairs of dangling edges of the same color. At the same way we successively cancel the dipole of type 1 formed by the vertices $9 a$ and $13 a$, the dipole of type 1 formed by the vertices $11 a$ and $12 a$, the dipole of type 1 formed by the vertices $13 b$ and $14 b$, the dipole of type 1 formed by the vertices $3 a$ and $5 a$, the dipole of type 1 formed by the vertices $12 b$ and $15 b$, the induced dipole of type 2 formed by the vertices $7 a$ and $8 a$, the dipole of type 1 formed by the vertices $6 b$ and $9 b$, the dipole of type 1 formed by the vertices $1 a$ and $2 a$, the induced dipole of type 2 formed by the vertices $7 b$ and $11 b$, the induced dipole of type 2 formed by the vertices $5 b$ and $8 b$, and the induced dipole of type 2 formed by the vertices $4 b$ and $6 a$. The resulting graph $\widetilde{G}^{(4,2)}$ has 36 vertices and it is contracted since $\widetilde{G}_{\widehat{i}}^{(4,2)}$ is connected for every $i \in \Delta_{6}$ (see Figure 2). Thus $\widetilde{G}^{(4,2)}$ is a crystallization which represents $\mathbb{S}^{4} \times \mathbb{S}^{2}$. We can directly calculate the numbers $g_{i j}, i, j \in \Delta_{6}$, of the 2-colored connected components of $\widetilde{G}^{(4,2)}$ :

$$
\begin{array}{lllllll}
g_{01}=9 & g_{02}=9 & g_{03}=9 & g_{04}=9 & g_{05}=8 & g_{06}=9 & g_{12}=10 \\
g_{13}=8 & g_{14}=10 & g_{15}=9 & g_{16}=10 & g_{23}=8 & g_{24}=10 & g_{25}=9 \\
g_{26}=10 & g_{34}=8 & g_{35}=9 & g_{36}=8 & g_{45}=9 & g_{46}=10 & g_{56}=9
\end{array} .
$$

Let us consider the cyclic permutation $\epsilon=\left(\epsilon_{0}, \epsilon_{1}, \ldots, \epsilon_{n}\right)=(0,1,2,4,6,5,3)$ of $\Delta_{6}$. Then $\sum_{i \in \mathbb{Z}_{7}} g_{\epsilon_{i} \epsilon_{i+1}}=66$, hence the graph $\widetilde{G}^{(4,2)}$ regularly embeds into the closed connected orientable surface $F$ whose Euler characteristic is

$$
\chi(F)=\sum_{i \in \mathbb{Z}_{7}} g_{\epsilon_{i} \epsilon_{i+1}}+\frac{(1-6)}{2} p=66-\frac{5}{2} 36=-24 .
$$

Then the genus of $F$ is $g(F)=(2-\chi(F)) / 2=13$, hence $g\left(\mathbb{S}^{4} \times \mathbb{S}^{2}\right) \leq 13$.

\section{A crystallization of $\mathbb{S}^{5} \times \mathbb{S}^{2}$}

Applying the construction described in Section 2 we can obtain a 8-colored graph $G^{(5,2)}$ with 84 vertices representing the manifold $\mathbb{S}^{5} \times \mathbb{S}^{2}$. According with the notation in Section 3 , the vertices $\bar{\delta}_{j}^{i}(k), i, j=1,2, k=1, \ldots,\left(\begin{array}{c}6 \\ 2\end{array}\right)=15$, are denoted in the following way: $\bar{\delta}_{1}^{1}(1)=1 a, \bar{\delta}_{1}^{2}(1)=1 b, \bar{\delta}_{2}^{1}(1)=1 c, \bar{\delta}_{2}^{2}(1)=1 d$, $\bar{\delta}_{1}^{1}(2)=2 a, \bar{\delta}_{1}^{2}(2)=2 b, \bar{\delta}_{2}^{1}(2)=2 c, \bar{\delta}_{2}^{2}(2)=2 d, \ldots, \bar{\delta}_{1}^{1}(15)=15 a, \bar{\delta}_{1}^{2}(15)=$ $15 b, \bar{\delta}_{2}^{1}(15)=15 c, \bar{\delta}_{2}^{2}(15)=15 d$; while the vertices $\overline{\bar{\delta}}_{j}^{i}(k), i, j=1,2, k=$ $1, \ldots,\left(\begin{array}{l}6 \\ 5\end{array}\right)=6$, are denoted as follows: $\overline{\bar{\delta}}_{1}^{1}(1)=16 a, \overline{\bar{\delta}}_{1}^{2}(1)=16 b, \overline{\bar{\delta}}_{2}^{1}(2)=16 c$, $\overline{\bar{\delta}}_{2}^{2}(1)=16 d, \ldots, \overline{\bar{\delta}}_{1}^{1}(6)=21 a, \overline{\bar{\delta}}_{1}^{2}(6)=21 b, \overline{\bar{\delta}}_{2}^{1}(6)=21 c, \overline{\bar{\delta}}_{2}^{2}(6)=21 d$. Then, according to steps (2) and (3) of the construction in Section 2, we join in pairs the vertices $1 a$ and $1 b$ (resp. $1 c$ and $1 d$ ), ., $15 a$ and $15 b$ (resp. $15 c$ and $15 d$ ), and the vertices $16 a$ and $16 c$ (resp. $16 b$ and $16 d$ ), ..,21a and $21 c$ (resp. $21 b$ and $21 d$ ) by 7 -colored edges. Finally, applying step (4), we join in pairs the 
vertices $11 a$ and $16 a$ (resp. $11 b, 11 c, 11 d$ with $16 b, 16 c, 16 d$ ), $\ldots, 15 a$ and $20 a$ (resp. $15 b, 15 c, 15 d$ with $20 b, 20 c, 20 d$ ) by 6 -colored edges. For the remaining vertices of $G^{(5,2)}$ we consider the edges as they are in the graphs $G^{(4,2)}$ and $G^{(5,1)}$, representing $\mathbb{S}^{4} \times \mathbb{S}^{2}$ and $\mathbb{S}^{5} \times \mathbb{S}^{1}$, respectively, according to step (5). Since the graph $G^{(5,2)}$ has a double symmetry we can draw only a part of it (see Figure 3). The whole graph can be obtained by reflecting twice the picture in Figure 3. We simplify the graph $G^{(5,2)}$ by cancelling 10 dipoles of type 1 and 10 dipoles of type 2 , obtaining a crystallization $\widetilde{G}^{(5,2)}$ for $\mathbb{S}^{5} \times \mathbb{S}^{2}$ with 44 vertices (see Figure 4). A direct calculation gives $g_{i j}=12$, for every $i, j \in \Delta_{7}$, hence the graph $\widetilde{G}^{(5,2)}$ regularly embeds into the closed connected orientable surface $F$ whose Euler characteristic is

$$
\chi(F)=\sum_{i \in \mathbb{Z}_{8}} g_{\epsilon_{i} \epsilon_{i+1}}+\frac{(1-7)}{2} p=96-\frac{6}{2} 44=-36
$$

for any cyclic permutation $\epsilon=\left(\epsilon_{0}, \epsilon_{1}, \ldots, \epsilon_{n}\right)$ of $\Delta_{7}$. Then the genus of $F$ is $g(F)=(2-\chi(F)) / 2=19$, hence $g\left(\mathbb{S}^{5} \times \mathbb{S}^{2}\right) \leq 19$.

Acknowledgements. Work performed under the auspices of the GNSAGA of the CNR (National Research Council) of Italy and partially supported by the MIUR (Ministero dell'Istruzione, dell'Università e della Ricerca) of Italy within the project "Proprietà Geometriche delle Varietà Reali e Complesse", and by a research grant of the University of Modena and Reggio Emilia.

\section{References}

[1] J. Bracho and L. Montejano, The combinatorics of colored triangulations of manifolds, Geom. Dedicata 22 (1987), no. 3, 303-328.

[2] M. R. Casali and C. Gagliardi, Classifying PL 5-manifolds up to regular genus seven, Proc. Amer. Math. Soc. 120 (1994), no. 1, 275-283.

[3] A. Cavicchioli, A combinatorial characterization of $S^{3} \times S^{1}$ among closed 4-manifolds, Proc. Amer. Math. Soc. 105 (1989), no. 4, 1008-1014.

[4] _ On the genus of smooth 4-manifolds, Trans. Amer. Math. Soc. 331 (1992), no. $1,203-214$.

[5] _ Manifold crystallization, in: Encyclopaedia of Mathematics, Kluwer Acad. Publ. (M. Hazewinkel, Editor-in-Chief; R. Hoksbergen, Coordinating Editor), Dordrecht, The Netherlands, 1998.

[6] A. Cavicchioli and F. Hegenbarth, On the determination of PL-manifolds by handles of lower dimension, Topology Appl. 53 (1993), no. 2, 111-118.

[7] A. Cavicchioli, D. Repovš, and A. B. Skopenkov, Open problems on graphs arising from geometric topology, Topology Appl. 84 (1998), no. 1-3, 207-226.

[8] P. Cristofori, On the genus of $\mathbb{S}^{m} \times \mathbb{S}^{n}$, J. Korean Math. Soc. 41 (2004), no. 3, 407-421.

[9] M. Ferri and C. Gagliardi, Crystallisation moves, Pacific J. Math. 100 (1982), no. 1, 85-103.

[10] M. Ferri, C. Gagliardi, and L. Grasselli, A graph-theoretical representation of PLmanifolds-a survey on crystallizations, Aequationes Math. 31 (1986), no. 2-3, 121-141.

[11] C. Gagliardi, Regular imbeddings of edge-coloured graphs, Geom. Dedicata 11 (1981), no. $4,397-414$.

[12] _ Extending the concept of genus to dimension n, Proc. Amer. Math. Soc. 81 (1981), no. 3, 473-481. 
[13] C. Gagliardi and L. Grasselli, Representing products of polyhedra by products of edgecolored graphs, J. Graph Theory 17 (1993), no. 5, 549-579.

[14] P. J. Hilton and S. Wylie, Homology Theory: An introduction to algebraic topology, Cambridge University Press, New York 1960

[15] M. Pezzana, Atlanti minimali di varietà differenziabili compatte, Boll. Un. Mat. Ital. 6 (1968), 749-752.

[16] Sulla struttura topologica delle varietá compatte, Atti Sem. Mat. Fis. Univ. Modena 23 (1974), no. 1, 269-277.

[17] C. Rourke and B. Sanderson, Introduction to Piecewise-Linear Topology, Ergebnisse der Mathematik und ihrer Grenzgebiete, Band 69. Springer-Verlag, New York-Heidelberg, 1972.

[18] A. Vince, n-graphs, Discrete Math. 72 (1988), no. 1-3, 367-380.

Dipartimento Di Matematica

Università di Modena e Reggio Emilia

Via Campi 213/B, 41100 Modena, Italy

E-mail address: fulvia.spaggiari@unimore.it 